\title{
The effects of transcranial direct current stimulation on metabolite changes at the anterior cingulate cortex in neuropathic pain: a pilot study
}

This article was published in the following Dove Press journal: Journal of Pain Research

\author{
Paradee Auvichayapat ${ }^{\prime}$ \\ Keattichai Keeratitanont ${ }^{2}$ \\ Taweesak Janyachareon ${ }^{3}$ \\ Narong Auvichayapat ${ }^{4}$ \\ 'Department of Physiology; \\ ${ }^{2}$ Department of Radiology; \\ ${ }^{3}$ Department of Physical Therapy; \\ ${ }^{4}$ Division of Pediatric Neurology, \\ Department of Pediatrics, Faculty of \\ Medicine, Khon Kaen University, Khon \\ Kaen, Thailand
}

Background: Neuropathic pain (NP) in individuals with spinal cord injury (SCI) is both common and highly refractory to treatment. Primary motor cortex stimulation can relieve pain by interrupting the transmission of noxious information of descending pain modulatory systems including the anterior cingulate cortex (ACC). Previous research has shown that transcranial direct current stimulation (tDCS) can produce pain relief in individuals with NP. However, the underlying mechanisms for these effects are not yet understood. Research findings suggest the possibility that changes in brain metabolite concentrations produced by tDCS might explain some of these effects. For example, previous research has shown that SCI-related NP is associated with elevated levels of glutamine combined glutamate (Glx) per creatine $(\mathrm{Glx} / \mathrm{Cr}$ ). In addition, decreased N-acetylaspartate (NAA) has been observed in the ACC in individuals with chronic pain. Methods: We used magnetic resonance spectroscopy (MRS) to study changes in NAA and Glx levels in the ACC after tDCS treatment. Ten patients with SCI with NP were given five daily anodal tDCS sessions, and an MRS evaluation was performed before and after treatment. Results: The results showed treatment-related reductions in pain, and increases in both $\mathrm{Glx} / \mathrm{Cr}$ and $\mathrm{NAA} / \mathrm{Cr}$ in the ACC. The observed increase in NAA/Cr is consistent with the possibility that tDCS improves the descending pain modulation system by increasing the neuronal activity in the ACC.

Conclusion: The findings suggest the possibility that tDCS's beneficial effects on neuropathic pain may be due, at least in part, to the changes it produces in $\mathrm{Glx} / \mathrm{Cr}$ and NAA/Cr levels in the ACC. Additional research with larger samples sizes and a control group to evaluate this possibility is warranted.

Keywords: magnetic resonance spectroscopy, neuropathic pain, spinal cord injury, transcranial direct current stimulation, anterior cingulate cortex

\section{Introduction}

Neuropathic pain (NP) from spinal cord injury (SCI) is both common and highly refractory to treatment. ${ }^{1}$ Thus, there continues to be a significant need to develop and identify effective treatments for SCI-related NP. A number of studies have shown promising beneficial effects of transcranial direct current stimulation (tDCS) in patients with NP. ${ }^{2}$ The beneficial effects of tDCS on NP may be related to the upregulation of motor cortex excitability in descending pain-modulating areas, such as the thalamus and anterior cingulate cortex. ${ }^{3}$ However, the mechanisms underlying the analgesic effects of tDCS are not yet fully understood.
Correspondence: Narong Auvichayapat Division of Pediatric Neurology,

Department of Pediatrics, Faculty of Medicine, Khon Kaen University, Khon Kaen, 40002, Thailand

Tel +6689622 2624

Email anaron@kku.ac.th 
Proton magnetic resonance spectroscopy (1H-MRS) is a noninvasive brain imaging method that can assess the concentrations of specific metabolites in different brain regions, including $\mathrm{N}$-acetylaspartate (NAA), choline (Cho), creatine (Cr), myo-inositol (mI), glutamine (Gln) combined glutamate (Glu), and gamma-aminobutyric acid. Concentration changes of these metabolites are associated with numerous neurological diseases and symptoms, including SCI-related NP. ${ }^{4}$

The anterior cingulate cortex (ACC) is thought to be involved in pain modulation. ${ }^{5}$ It is one of the areas most commonly activated by noxious stimuli in human brain and may influence affect-related nociceptive connectivity in other regions. ${ }^{6,7}$ Consistent with this idea, research has shown that development of neuropathic pain is associated with increased cerebral blood flow in the ACC. ${ }^{8}$

In addition, measures of metabolite concentrations in the ACC have been shown to be related to pain. ${ }^{9,10}$ For example, pain intensity has been shown to be associated with higher Glu and low NAA levels. ${ }^{11,12}$ Elevated levels of Cho have also been found in ACC in individuals with pelvic pain syndrome, relative to healthy subjects. ${ }^{13}$ Based on these findings, it can be hypothesized that alterations in brain metabolites, specifically elevations in concentration of Gln combined Glu (Glx), NAA, and Cho in the ACC are associated with elevations in pain.

Along these lines, it would also be reasonable to hypothesize that the analgesic effects of tDCS in SCI-related NP may be related to the effects of tDCS on metabolite concentrations. Preliminary studies support this idea. ${ }^{10,14}$ For example, changes of $\mathrm{mI}$ concentrations have been found following a single session of tDCS. ${ }^{14}$ Another preliminary study showed decreases in NAA/Cr in the primary motor cortex in nine individuals with chronic pelvic pain patients after 10 sessions of tDCS. ${ }^{10}$ However, to the best of our knowledge, there is no study that has examined brain metabolite changes in the ACC following tDCS.

Given these considerations, the purpose of this pilot study was to determine if there may be changes to brain metabolites in the ACC with tDCS. Ten adults with SCIrelated NP were given five sessions of tDCS and the effects of this treatment were measured on both pain intensity and metabolite concentrations in the ACC. We hypothesized that tDCS treatment would decrease pain intensity and also increase $\mathrm{Glx} / \mathrm{Cr}, \mathrm{NAA} / \mathrm{Cr}$, and $\mathrm{Cho} / \mathrm{Cr}$ concentrations in the ACC. We also hypothesized that treatment-related changes in pain intensity would be associated with treatment-related changes in the metabolite concentrations.

\section{Materials and methods}

\section{Study design}

This pilot study was performed over 5 weeks consisting of: 1) a 1-week baseline assessment; 2) 5 consecutive days of $2 \mathrm{~mA}$ anodal tDCS for 20 minutes; and 3) a 3-week followup period. Measures were administered during baseline and during treatment, and then again weekly during the followup period. Participants were asked to continue their routine analgesic medication regimen throughout the duration of the 5-week study.

\section{Participant recruitment and informed consent}

Study participants were recruited via advertisements placed in the inpatient unit of medical rehabilitation, Faculty of Medicine, Khon Kaen University, Thailand. The study procedures were described to eligible patients who expressed an interest in participating in the study by clinic physicians. Study inclusion criteria were: 1) being right handed and having a history of a traumatic spinal cord injury due to an accident, a gunshot, or disease; 2) bilateral pain that had the same intensity on both sides of the body with an average pain intensity rating before tDCS treatment of 4 or greater, as measured by a $0-10$ numerical rating scale ( $0=$ "no pain;" $10=$ "worst pain that you could imagine"); 3 ) presenting with a pain problem that was refractory to medications, including tricyclic antidepressants, antiepileptic drugs, and/or opioids (ie, pain that does not respond to at least two of these drugs supplied in adequate doses for 6 months); 4) no evidence of progressive neurological disease or other secondary conditions that could impact pain; and 5) no alternative treatment for pain (herbal remedies and other alternative therapies) used in the previous month. The exclusion criteria were: 1) a diagnosis of syringomyelia; 2) current or history of drug abuse or addiction; 3) current psychiatric disorder such as schizophrenia (per medical record), depression (as indicated by a score of 16 or greater on the Beck Depression Inventory, or severe anxiety as indicated by a score of 39 or lower-lower scores indicated greater anxiety - on the Anxiety scale of the Symptom Checklist-90 (Department of Mental Health, 2011a); 4) significant cognitive deficits as indicated by a score of 22 or lower on the Thai Mental State Examination (Department of Mental Health, 2011b); 5) history of loss of consciousness or post-traumatic amnesia at the time of injury; 6) skull defects that would interfere with tDCS treatment; and 7) contraindications for undergoing an MRI scan (ie, patients 
who have a heart pacemaker, a metallic foreign body in an eye, an aneurysm clip in the brain, or severe claustrophobia). The study was conducted in accordance with the Declaration of Helsinki and was approved by the Ethics Committee of Khon Kaen University (identifier number: HE561320). Written informed consent was obtained from all patients before participation. This protocol had been approved for registration at Thai Clinical Trials Registry (TCTR; identification number: TCTR20170822003).

\section{Sample size calculation}

The number of subjects needed in this pilot study to have adequate power to detect significant pre- to post-treatment changes in pain intensity and metabolite concentrations was determined based on the findings from the previous study, which found that a single tDCS induced a significant increase in the concentration of $\mathrm{mI}$ at 30 minutes after stimulation offset $(141.5 \% \pm 16.7 \%$, $P<0.001) .{ }^{14}$ If tDCS had a similar effect on the outcomes as in the current study, 10 participants would provide a power of 0.90 to detect this as significant with an alpha of 0.05 .

\section{Measures}

The primary outcomes of this study were pain intensity and levels of brain metabolites in the ACC.

\section{Pain intensity}

Average pain intensity was assessed using 0-10 numerical rating scale (NRS). Participants were asked to provide ratings of average pain in the past 24 hours for 7 days during the 1 -week baseline period in a daily diary. These seven days rating were then averaged into a single composite score of baseline average pain intensity. The participants were also asked to rate average pain in the past 24 hours before and immediately after each tDCS session ( 5 consecutive days). For purposes of data analysis, the outcome rating obtained after each session was used to test the effects of treatment on average pain intensity over time. The ratings obtained before each session were used to determine if there was any loss of treatment benefit since the last session (ie, by comparing them to the rating obtained just after the previous session). 24-hour recall ratings of average pain intensity were then also obtained at 1-week, 2-week, and 3-week post-treatment, via telephone interview. 0-10 NRSs have a great deal of evidence supporting their reliability and validity as measures of pain intensity, and are recommended as the measure of pain intensity with the most strengths and fewest weaknesses by the Initiative on Methods, Measurement, and Pain Assessment in Clinical Trials consensus group. ${ }^{15}$

\section{Brain metabolite level assessment}

A 1-hour MRS assessment was used to measure baseline and post-treatment levels of NAA, Glx, Cho, $\mathrm{mI}$, and $\mathrm{Cr}$ in the ACC, using a Philips Achieva 3.0T (Philips Healthcare, Best, Netherlands) running Release 2.6.3.3 MR Workspace software. The participants were asked to lie still for $\sim 60$ minutes while the data were collected. Single-voxel proton MR spectra were acquired and quantified with an LC Model to determine metabolite concentration ratios. The MRS voxels $(2 \times 2 \times 2 \mathrm{~cm})$ were positioned on the coronal, sagittal, and axial images from the ACC (Figure 1). The spectra were acquired using a point resolved spectroscopy sequence with a repetition time of 2 seconds, short echo time of $35 \mathrm{~ms}$, spectral width of $2,000 \mathrm{~Hz}, 1,024$ time points, partial water suppression. Shimming was performed using manufacturersupplied shimming procedures. Analysis of the metabolite concentration was performed using an LC Model (Stephen Provencher Inc., Oakville, ON, Canada). Levels of NAA, Glx, $\mathrm{mI}, \mathrm{Cr}$, and Cho were analyzed by fitting a linear combination of a basis set of metabolite model spectra to the data. Analyzing spectrum was set from $3.8 \mathrm{ppm}$ down to $0.2 \mathrm{ppm}$ with no eddy-current correction and water scaling. The metabolite concentrations were expressed as $\mathrm{mM}$ and ratios relative to $\mathrm{Cr}$ peak. The metabolite concentrations and metabolite to $\mathrm{Cr}$ ratios were determined in these two spectra for each subject.

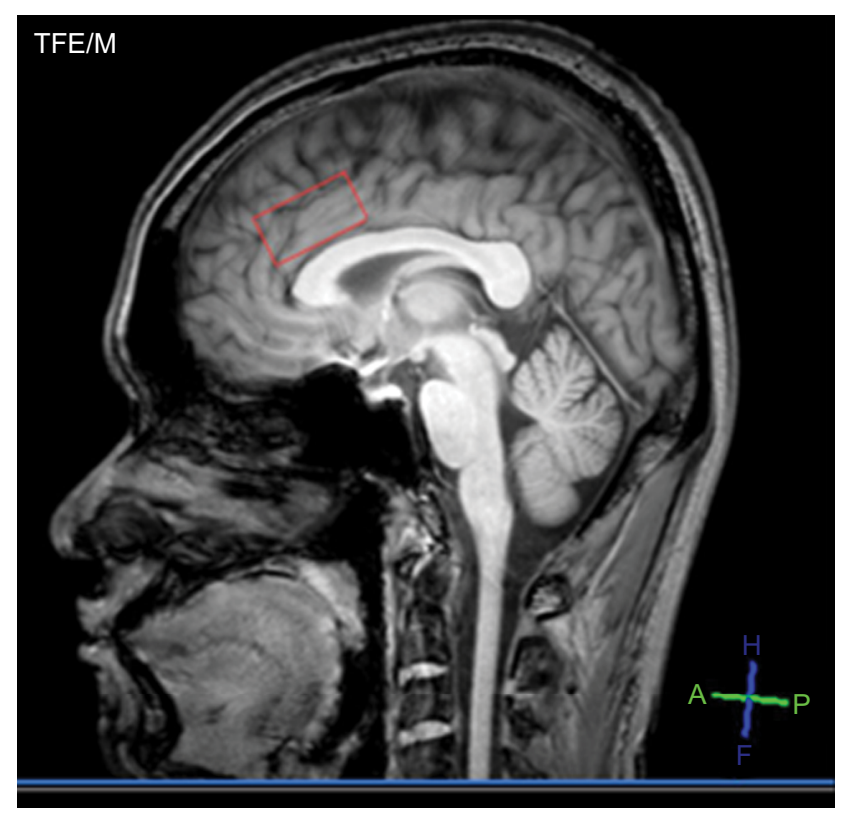

Figure I Areas of interest, ie, anterior cingulate cortex.

Note: The red box indicates the location of a single voxel in the anterior cingulate cortex.

Abbreviations: A, anterior; $f$, feet; $h$, head; $p$, posterior; TEF/M, turbo field echo modulus type. 
tDCS was applied via a pair of $0.9 \% \mathrm{NaCl}$-soaked surface sponge electrodes $\left(35 \mathrm{~cm}^{2}\right)$ and delivered through a batterydriven power supply. The constant current stimulator had a maximum output of $10 \mathrm{~mA}$ (Soterix Medical, Model 1224-B, New York, NY, USA). The anode electrode was placed over the left M1, which was located by single-pulse transcranial magnetic stimulation. Direct current of $2 \mathrm{~mA}$ for 20 minutes was administered once a day for 5 consecutive days during the treatment period. The cathode (reference) electrode was placed on the shoulder contralateral to the anode. This stimulation paradigm was chosen because it has demonstrated that extracephalic montages, eg, contralateral shoulder might create larger total current densities in deeper brain regions such as the ACC as compared to an equivalent cephalic montage in chronic pain populations, and provides a broad pattern of stimulation to motor, somatosensory, and frontal cortices implicated in pain sensitivity. ${ }^{15,16}$

\section{Adverse events and safety}

The participants were asked to report any adverse events as well as other signs and symptoms immediately after each of the stimulation sessions. Participants were also closely observed by physicians during and just after the study sessions.

\section{Data analysis}

Data were analyzed using the Stata software, version 10.0 (StataCorp LP, College Station, TX, USA) and outcomes were reported as means and standard deviations (mean \pm $\mathrm{SD})$. Changes in pain intensity over the course of treatment and through follow-up were evaluated using repeated measure ANOVAs. Maintenance of gains in improvements of pain intensity from one treatment session to the next were evaluated using paired sample $t$-tests. The nonparametric Wilcoxon rank sum test was used to evaluate differences of the metabolite changes between baseline and after completion of five daily tDCS sessions (metabolite changes on day 5), because of the relatively low sample size as well as the fact that the metabolite scores did not have normal distributions, as determined by the Shapiro-Wilk test. Finally, we examined the associations between metabolite levels and pain intensity by computing correlation coefficients between pre- to posttreatment changes in the measurements of these domains.

\section{Results}

\section{Sample description}

A total of ten patients with SCI and NP were enrolled into the study between December 2013 and July 2014. They had a mean age of 32.7 years $(\mathrm{SD}=6.88)$. No participants were screened out of the study due to psychological factors (ie, diagnosis of schizophrenia or meeting cutoff scores for depression or anxiety) or cognitive dysfunction. There was no change in pharmacological treatment during the tDCS treatment in any participants. The participants' demographic and SCI history characteristics, and baseline average pain intensity, are presented in Table 1.

\section{Change in pain intensity}

The repeated measures ANOVA with pain intensity as the dependent variable and time (baseline, day 1 to 5, 1-week, 2-week, and 3-week follow-up) as a within-subjects independent variable revealed a significant main effect for time with a large effect size $\left(F(8,9)=7.03 ; P<0.001, \eta^{2}=0.339\right)$. In order to interpret the significant effect of time, we performed post hoc analyses of the time factor. These revealed a significant decrease in pain intensity from baseline-to postsession at day $2(0.613,95 \% \mathrm{CI}=0.002-1.224 ; P=0.049)$, day $3(1.413,95 \% \mathrm{CI}=0.113-2.713 ; P=0.036)$, day 4 $(1.613,95 \% \mathrm{CI}=0.155-3.071 ; P=0.034)$, day $5(2.213,95 \%$ $\mathrm{CI}=0.836-3.570 ; P=0.005), 1$-week follow-up $(2.213,95 \%$ $\mathrm{CI}=0.735-3.691 ; P=0.008)$, and 2-week follow-up (1.613, 95\% CI=0.043-3.183; $P=0.045)$, respectively. A return to baseline level in average pain intensity began at the 2 -week follow-up point, and the 3 -week rating was not significantly different from the baseline rating $(0.213,95 \% \mathrm{CI}=-0.115-$ $0.541, P=0.175$ ) (see Figure 2).

\section{Change in metabolite concentrations}

For all individuals, MRS data were acquired from the ACC. The data analysis showed a statistical increase in NAA compared with baseline $(P=0.005)$ and a significant increase in Glx $(P=0.005)$. However, there was no significant change in $\mathrm{mI}$ or Cho, compared with baseline $(P=0.574$ and 0.138 , respectively) (Table 2). With regard to effect sizes (Cohen's d), the pre- to post-treatment changes in NAA/Cr, Glx/Cr, Cho/Cr, and $\mathrm{mI} / \mathrm{Cr}$ were 0.97 (large), 1.26 (large), 0.21 (weak), and 0.25 (weak), respectively (see Figure 3).

\section{Association between pain intensity and metabolite changes}

Wilcoxon rank sum tests revealed significant association between improvements in pain and an increase in both $\mathrm{Glx} / \mathrm{Cr}(r=0.75, P=0.013)$ and NAA $(r=0.61, P=0.049)$. However, there were weak (and nonsignificant) associations between improvements in pain and changes in $\mathrm{mI} /$ $\mathrm{Cr}(r=0.20, P=0.587)$ and $\mathrm{Cho} / \mathrm{Cr}(r=-0.11, P=0.759)$ (Figure 4). 


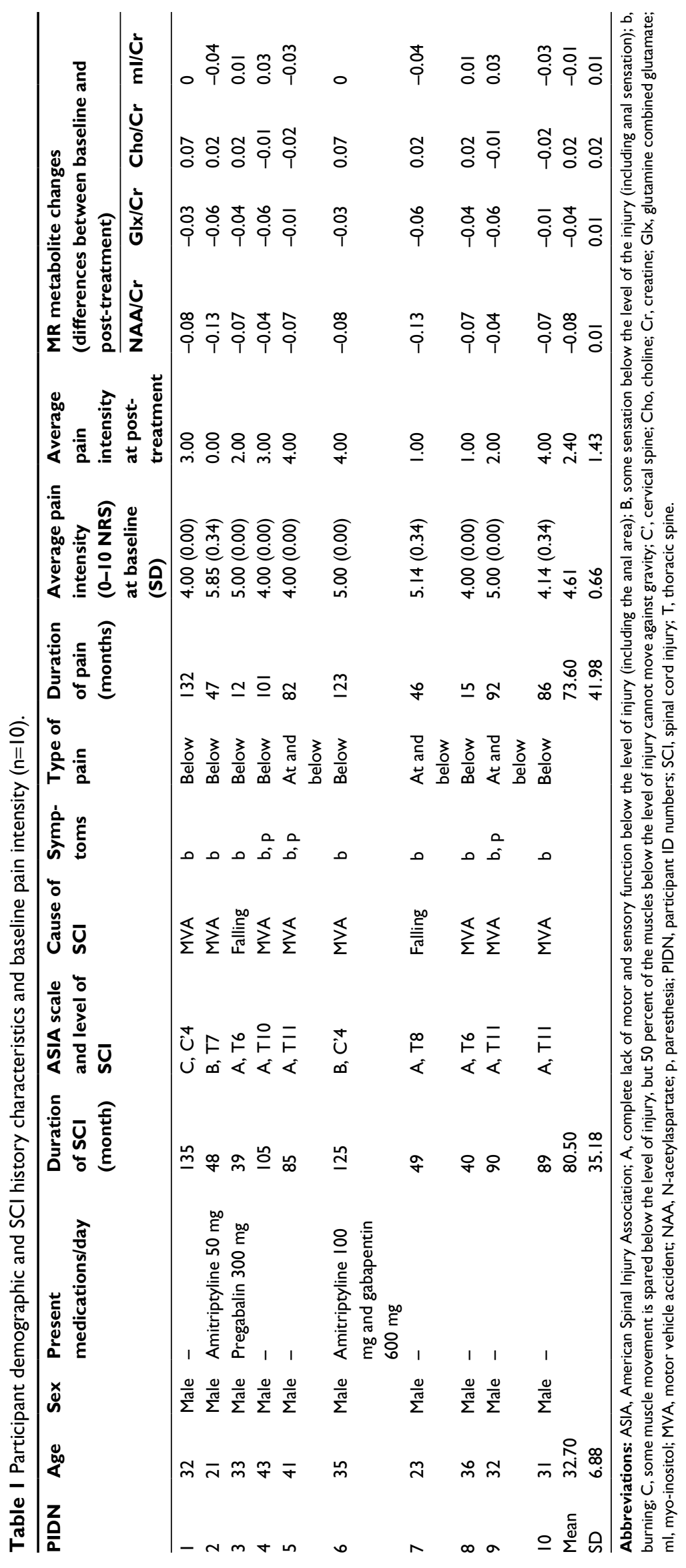




\section{Adverse events and safety}

In respect to adverse effects, all of the participants tolerated the stimulation procedure well, and none reported any discomfort during stimulation. However, four participants evidenced erythematous rashes under the cathodal electrode placed over their right shoulders during treatment. All of these rashes resolved within 2 hours of follow-up.

\section{Discussion}

The present findings showed statistically significant pre- to post-treatment improvements in pain intensity as well as significant pre- to post-treatment increases in NAA/Cr and $\mathrm{Glx} / \mathrm{Cr}$ in the ACC. However no significant changes in $\mathrm{mI} /$ $\mathrm{Cr}$ and Cho were found.

The perception of nociceptive input is mediated through the spinothalamic pathway and then modulated and processed by higher cortical brain regions, including the ACC..$^{5,7}$

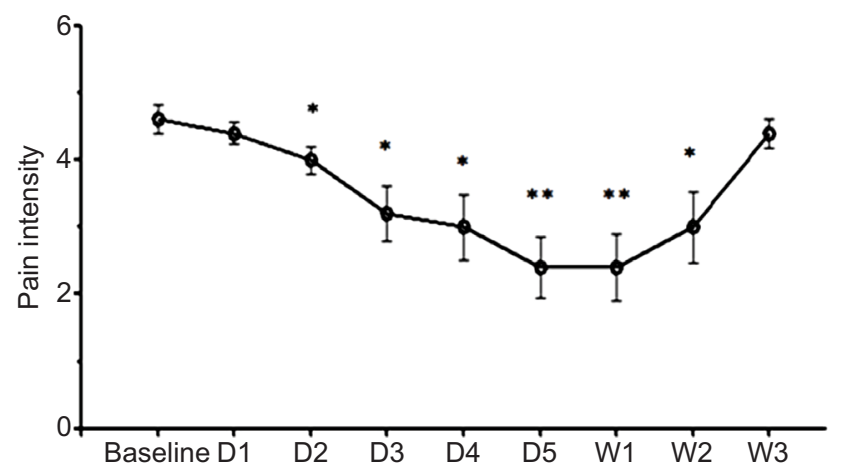

Figure 2 Effects of tDCS treatments on pain intensity.

Notes: Data are presented as the means of NRS at baseline (before tDCS) and after various time points after treatment on DI, D2, D3, D4, D5, and I, 2, 3 weeks after 5 consecutive days of tDCS. Vertical lines represent SEM. Significant differences from baseline are represented by $* P<0.05$ and $* * P<0.01$.

Abbreviations: $D$, day; NRS, 0-10 numerical rating scale; tDCS, transcranial direct current stimulation; $W$, week.
Depending on both the magnitude of the nociceptive input as well as the factors that influence the processing of that input (eg, previous learning, focus of attention, etc), the brain will create (or not) the experience of pain. In the special case of SCI-related NP pain, damage to the spinothalamic tract associated with the injury can contribute to the development and maintenance of NP after SCI, interfering with natural pain inhibitory processes, and resulting in highly refractory - and often very severe - NP in these individuals. ${ }^{17}$

NAA is an amino acid derivative which is found exclusively in central nervous system (CNS) neurons. It is considered as a marker of global neuronal health and attenuation. ${ }^{18}$ Lower levels of this neurotransmitter are found in a variety of CNS disorders that cause neuronal cell destruction, such as multiple sclerosis, Alzheimer's disease, and high-grade brain tumor. ${ }^{19,20}$ Moreover relatively low levels of NAA are also found in some chronic conditions that have chronic pain

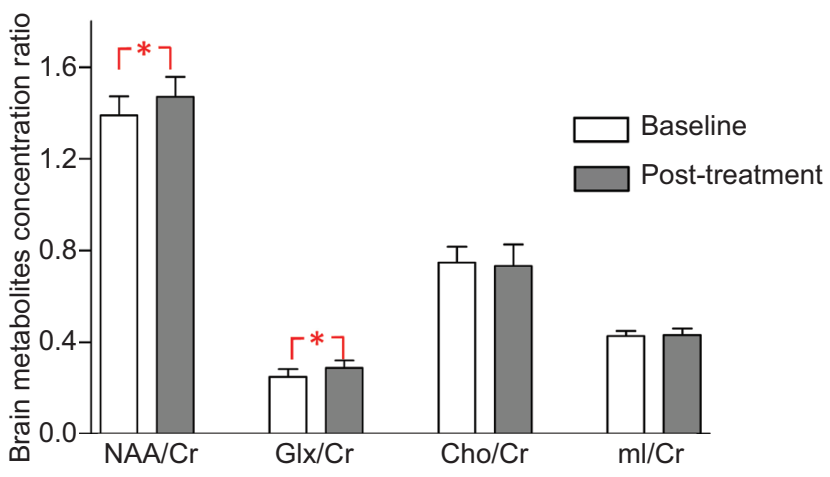

Figure 3 Metabolite changes in the anterior cingulate cortex. Data are presented as mean of brain metabolites compared between baseline and post-tDCS treatment. Notes: Vertical lines represent SD. *Represents significant difference $P<0.01$.

Abbreviations: Cho, choline; $\mathrm{Cr}$, creatine; Glx, glutamine combined glutamate; $\mathrm{ml}$, myo-inositol; NAA, N-acetylaspartate; tDCS, transcranial direct current stimulation.

Table 2 Differences between baseline and post-treatment of brain metabolites in the anterior cingulate cortex

\begin{tabular}{|c|c|c|c|c|c|c|c|}
\hline \multirow[t]{3}{*}{$\begin{array}{l}\text { Brain } \\
\text { metabolites }\end{array}$} & \multirow[t]{3}{*}{ Time } & \multirow[t]{3}{*}{$\begin{array}{l}\text { Brain metabolites } \\
\text { mean (SD) }\end{array}$} & \multicolumn{4}{|c|}{$\begin{array}{l}\text { Paired differences between baseline } \\
\text { and post-treatment }\end{array}$} & \multirow[t]{3}{*}{$P$-value } \\
\hline & & & \multirow[t]{2}{*}{ Mean } & \multirow[t]{2}{*}{ SD } & \multicolumn{2}{|c|}{$\begin{array}{l}95 \% \text { confidence interval } \\
\text { of the difference }\end{array}$} & \\
\hline & & & & & Lower & Upper & \\
\hline \multirow[t]{2}{*}{ NAA/Cr } & Baseline & $1.392(0.08)$ & & & & & \\
\hline & Post-treatment & $1.470(0.09)$ & -0.078 & 0.031 & -0.100 & -0.056 & $<0.001$ \\
\hline \multirow[t]{2}{*}{ GIx/Cr } & Baseline & $0.248(0.03)$ & & & & & \\
\hline & Post-treatment & $0.288(0.03)$ & -0.040 & 0.020 & -0.054 & -0.0256 & $<0.001$ \\
\hline \multirow[t]{2}{*}{ Cho/Cr } & Baseline & $0.748(0.07)$ & & & & & \\
\hline & Post-treatment & $0.732(0.09)$ & 0.016 & 0.033 & -0.008 & 0.040 & 0.160 \\
\hline \multirow[t]{2}{*}{$\mathrm{ml} / \mathrm{Cr}$} & Baseline & $0.426(0.02)$ & & & & & \\
\hline & Post-treatment & $0.432(0.03)$ & -0.006 & 0.027 & -0.025 & 0.0130 & 0.502 \\
\hline
\end{tabular}

Abbreviations: Cho, choline; Cr, creatine; Glx, glutamine combined glutamate; ml, myo-inositol; NAA, N-acetylaspartate. 


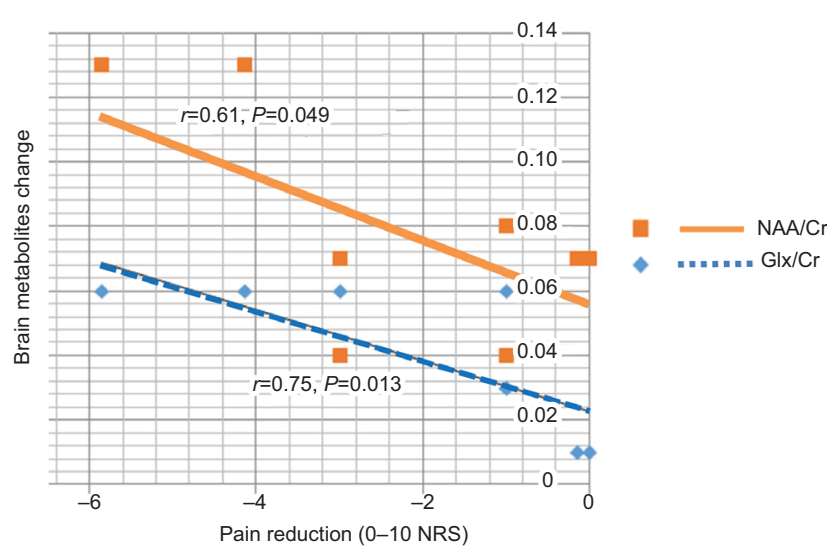

Figure 4 Correlation between pain reduction and brain metabolites change. Abbreviations: Cho, choline; $\mathrm{Cr}$, creatine; Glx, glutamine combined glutamate; NAA, N-acetylaspartate.

components, including diabetic painful neuropathy, chronic neuropathic pain, and chronic low back pain. ${ }^{11,12,21}$ Thus, it is reasonable to hypothesize that individuals with chronic pain might have lower concentrations of NAA in the ACC, relative to healthy participants. ${ }^{11,12}$ Consistent with a role for this metabolite in chronic pain, research has found a positive correlation between NAA levels and pain thresholds. ${ }^{10}$ However, our study found that tDCS could increase concentration levels of NAA. This may have been due to the possibility that individuals with chronic pain usually have low levels of NAA in the ACC. Thus, it might result in increases in the concentration of NAA, thereby causing decreases in pain intensity. Overall, the findings are consistent with the possibility that NAA plays an important role in pain modulation in the ACC.

There is increasing interest in the role of Glx in human neurophysiology, including pain perception. ${ }^{22} \mathrm{Glu}$ is compartmentalized in neurons, while Gln is compartmentalized in glia cells. Glu is the most abundant excitatory neurotransmitter in the human brain, with a role in synapsis activity, where it is released from presynaptic cells, and then binds to postsynaptic receptors to induce activation.

Research showing a positive association between general ACC activity and chronic NP supports the role of the ACC as one of key components of the pain matrix. ${ }^{5,7,8}$ Consistent with the notion of Glu as an activating neurotransmitter, research has shown higher concentrations of Glu in the ACC of individuals with chronic pain, relative to healthy subjects. ${ }^{11,12}$ Such increases could be associated with the brain's attempts to activate inhibition processes. Our study found increases in glutamate levels in the ACC after tDCS treatment, and that this elevation was associated with pain reduction.
Cho is thought to be a phospholipid in cell membranes. Thus, an increase in Cho might be indicative of cell membrane synthesis and increases in cellularity. ${ }^{18}$ Prior study has shown an increase of Cho in glia cell tumors, suggesting that Cho concentration correlates with increase cellularity. ${ }^{23}$ There has been very little research that has examined the role of Cho in pain perception, although one study found an elevation in Cho concentrations in the ACC in a sample of individuals with spinal cord injury and chronic pain. ${ }^{9}$ However, we did not find a significant change in Cho concentrations in the ACC with tDCS treatment.

Interfering with natural pain inhibitory processes is likely influenced by the release and uptake of brain metabolites. For example, $\mathrm{mI}$ is a sugar-like molecule found in brain glial cells that can be viewed as a marker of glial proliferation, given that the osmotic balance in the tissue is preserved by regulation of $\mathrm{mI}$ transport across the plasma membrane. ${ }^{24}$ Again, however, we did not find any significant change in $\mathrm{mI} / \mathrm{Cr}$ in the ACC after tDCS treatment.

There are multiple possible reasons for our null findings in respect to $\mathrm{Cho}$ and $\mathrm{mI} / \mathrm{Cr}$, including the small sample size of the current study (ie, lack of power to detect an effect) and very low levels of Cho in our sample at baseline (ie, which may make it challenging to enlist enough of a response). It is also possible that tDCS has no impact on the release of this metabolite. Given the potential role that Cho plays in pain, however, it is probably too early to rule out Cho as a potential mechanism for the benefits of tDCS and other pain treatments.

\section{Limitations}

The present study had some limitations that should be considered when interpreting the results. First, even though we powered the study to be able to detect large effects, the study had limited power for detecting moderate or weak effects. Thus, we may have been unable to detect significant effects in the current sample that in fact exist in the population. Therefore, the findings presented here should be viewed as tentative and in need of replication, ideally in studies using larger samples. Second, we did not include a placebo or sham treatment condition. Thus, we were not able to determine the extent to which the effects found on pain intensity and the metabolites that showed change were due to time effects of placebo effects. Future research including a control condition would be necessary to evaluate these effects. Finally, the tDCS intervention consisted of 5 consecutive days of 20-minute tDCS. This standard tDCS protocol might not be enough to remodel thalamic neurons or glia cells. Further 
study using a "higher dose" (ie, more sessions) of tDCS to determine if this has a larger impact on changes in pain and metabolite concentrations would be useful.

\section{Summary and conclusions}

Despite the study's limitations, the findings provide important new information regarding the beneficial effects of tDCS on neuropathic pain, and the potential that these effects may be explained by tDCS-related changes in metabolite concentrations. Specifically, the findings suggest that tDCS increases $\mathrm{NAA} / \mathrm{Cr}$, and $\mathrm{Glx} / \mathrm{Cr}$ in individuals with SCI-related NP. The increases observed in $\mathrm{Glx} / \mathrm{Cr}$ are consistent with the possibility that $\mathrm{tDCS}$ improves the efficacy of the descending pain modulation system in the ACC. The findings here indicate that additional research with a larger sample size and perhaps an even larger dose of tDCS treatment is warranted.

\section{Acknowledgments}

We thank Professor Mark P Jensen, University of Washington, for his guidance, valuable suggestions, and English language editing. We also thank the MRS radiologist Professor Jureerat Tammaroj, the MRS technician Wuttisak Boonphongsathian, the Physical Medicine and Rehabilitation physician, Professor Preeda Arayawichanon, the research staff Niran Ngernyam, Benchaporn Aneksan, and Anuwat Amatachaya, and the statistical analysis and graph design staff Au Auvichayapat and Vichaya Auvichayapat for their invaluable assistance. This work was supported by an invitation research grant, Faculty of Medicine, Khon Kaen University, Thailand (grant number IN61106), a grant of the National Research Council of Thailand.

\section{Disclosure}

The authors report no conflicts of interest in this work.

\section{References}

1. Nicholson BD. Evaluation and treatment of central pain syndromes. Neurology. 2004;62(5 Suppl 2):S30-S36.

2. Ngernyam N, Jensen MP, Arayawichanon P, et al. The effects of transcranial direct current stimulation in patients with neuropathic pain from spinal cord injury. Clin Neurophysiol. 2015;126(2):382-390.

3. Peyron R, Faillenot I, Mertens P, Laurent B, Garcia-Larrea L. Motor cortex stimulation in neuropathic pain. Correlations between analgesic effect and hemodynamic changes in the brain. A PET study. Neuroimage. 2007;34(1):310-321.

4. Gustin SM, Wrigley PJ, Youssef AM, et al. Thalamic activity and biochemical changes in individuals with neuropathic pain after spinal cord injury. Pain. 2014;155(5):1027-1036.

5. Bentley LD, Duarte RV, Furlong PL, Ashford RL, Raphael JH. Brain activity modifications following spinal cord stimulation for chronic neuropathic pain: A systematic review. Eur J Pain. 2016;20(4):499-511.
6. Apkarian AV, Bushnell MC, Treede RD, Zubieta JK. Human brain mechanisms of pain perception and regulation in health and disease. Eur J Pain. 2005;9(4):463-463.

7. Tsuda M, Koga K, Chen T, Zhuo M. Neuronal and microglial mechanisms for neuropathic pain in the spinal dorsal horn and anterior cingulate cortex. J Neurochem. 2017;141(4):486-498.

8. Watanabe K, Hirano S, Kojima K, et al. Altered cerebral blood flow in the anterior cingulate cortex is associated with neuropathic pain. J Neurol Neurosurg Psychiatry: 2018;pii:jnnp-2017-316601.

9. Widerström-Noga E, Pattany PM, Cruz-Almeida Y, et al. Metabolite concentrations in the anterior cingulate cortex predict high neuropathic pain impact after spinal cord injury. Pain. 2013;154(2): 204-212.

10. Simis M, Reidler JS, Duarte Macea D, et al. Investigation of central nervous system dysfunction in chronic pelvic pain using magnetic resonance spectroscopy and noninvasive brain stimulation. Pain Pract. 2015;15(5):423-432.

11. Ito $\mathrm{T}$, Tanaka-Mizuno $\mathrm{S}$, Iwashita $\mathrm{N}$, et al. Proton magnetic resonance spectroscopy assessment of metabolite status of the anterior cingulate cortex in chronic pain patients and healthy controls. J Pain Res. 2017;10:287-293.

12. Kameda T, Fukui S, Tominaga R, et al. Brain metabolite changes in the anterior cingulate cortex of chronic low back pain patients and correlations between metabolites and psychological state. Clin J Pain. 2018;34(7):657-663.

13. Harper DE, Ichesco E, Schrepf A, et al. Relationships between brain metabolite levels, functional connectivity, and negative mood in urologic chronic pelvic pain syndrome patients compared to controls: a MAPP research network study. Neuroimage Clin. 2018;17:570-578.

14. Rango M, Cogiamanian F, Marceglia S, et al. Myoinositol content in the human brain is modified by transcranial direct current stimulation in a matter of minutes: a 1H-MRS study. Magn Reson Med. 2008;60(4):782-789.

15. Turk DC, Dworkin RH, Burke LB, et al. Developing patient-reported outcome measures for pain clinical trials: IMMPACT recommendations. Pain. 2006;125(3):208-215.

16. Ahn H, Woods AJ, Kunik ME, et al. Efficacy of transcranial direct current stimulation over primary motor cortex (anode) and contralateral supraorbital area (cathode) on clinical pain severity and mobility performance in persons with knee osteoarthritis: An experimenter- and participant-blinded, randomized, sham-controlled pilot clinical study. Brain Stimul. 2017;10(5):902-909.

17. Widerström-Noga E, Cruz-Almeida Y, Felix ER, Pattany PM. Somatosensory phenotype is associated with thalamic metabolites and pain intensity after spinal cord injury. Pain. 2015;156(1):166-174.

18. Verma A, Kumar I, Verma N, Aggarwal P, Ojha R. Magnetic resonance spectroscopy - revisiting the biochemical and molecular milieu of brain tumors. BBA Clin. 2016;5:170-178.

19. Rigotti DJ, Inglese M, Gonen O. Whole-brain N-acetylaspartate as a surrogate marker of neuronal damage in diffuse neurologic disorders. Am J Neuroradiol. 2007;28(10):1843-1849.

20. Gonen O, Catalaa I, Babb JS, et al. Total brain N-acetylaspartate: a new measure of disease load in MS. Neurology. 2000;54(1):15-19.

21. Sorensen L, Siddall PJ, Trenell MI, Yue DK. Differences in metabolites in pain-processing brain regions in patients with diabetes and painful neuropathy. Diabetes Care. 2008;31(5):980-981.

22. Ramadan S, Lin A, Stanwell P. Glutamate and glutamine: a review of in vivo MRS in the human brain. NMR Biomed. 2013;26(12): $1630-1646$.

23. Chang L, McBride D, Miller BL, et al. Localized in vivo $1 \mathrm{H}$ magnetic resonance spectroscopy and in vitro analyses of heterogeneous brain tumors. J Neuroimaging. 1995;5(3):157-163.

24. Brand A, Richter-Landsberg C, Leibfritz D. Multinuclear NMR studies on the energy metabolism of glial and neuronal cells. Dev Neurosci. 1993;15(3-5):289-298. 
The Journal of Pain Research is an international, peer reviewed, open access, online journal that welcomes laboratory and clinical findings in the fields of pain research and the prevention and management of pain. Original research, reviews, symposium reports, hypothesis formation and commentaries are all considered for publication
The manuscript management system is completely online and includes a very quick and fair peer-review system, which is all easy to use. Visit http://www.dovepress.com/testimonials.php to read real quotes from published authors. 